Digital Press Social Sciences and Humanities

L'influence du genre à la transmission de la mémoire : Cas de Dora Bruder de Patrick Modiano

Fauzan Hanif

Proceeding of Conférence internationale sur le français 2018

Joesana Tjahjani, Merry Andriani, Sajarwa, Wening Udasmoro (eds) 


\title{
L'influence du genre à la transmission de la mémoire : Cas de Dora Bruder de Patrick Modiano
}

\author{
Fauzan Hanif \\ Universitas Gadjah Mada, Yogyakarta, Indonesia \\ e-mail : fauzan.hanif@mail.ugm.ac.id
}

\section{Résumé}

Lorsque des expériences culturelles sont stockées sous forme de mémoire d'une génération, ces souvenirs seront transmis de cette génération à l'autre. Surtout, les expériences traumatiques qui se traduisent par ce qu'on appelle la transmission de la mémoire. Dans le roman Dora Bruder, il existe une relation entre les récits traumatiques apportés par Patrick Modiano en tant qu'écrivain et le rôle de genre dans le processus de transmission de la mémoire. Le narrateur retrace la disparition d'une fille nommée Dora Bruder. Elle a perdu en 1941, lorsque les nazis occupaient la France. Cette recherche vise à découvrir la relation entre le jeu de genre et les narrations traumatiques dans ce roman en exploitant le concept de genre et de post mémoire. Sur la base des données obtenues, on apprend que le jeu de genre influence le processus de transmission de la mémoire. Ainsi, par cette manière Modiano décrit le message qu'on apprend que celui est dominé par d'absence. Partant de la sensation d'absence, il transmit consécutivement le vide, le doute, et aussi l'espérance de qu'un jour, il pourrait fixer cette absence par combler le trou profond causé par ce sentiment. Pour transmettre son message, Modiano utilise le mélange parmi des documents réels et factuels, ses imaginations de fiction, dont il manifeste sur l'histoire de quelqu'une, en racontant au point de vu de premier personnage. Il exploite cette manière pour s'identifier ; que quelqu'un ou quelqu'une, dans un épisode particulier de sa vie, a « les contextes partagés » avec l'autre.

\section{Mots-clés}

Dora Bruder, post-mémoire, transmission de la mémoire, genre, expérience traumatique

\begin{abstract}
Such cultural experiences have a possibility to be embedded in a memory of one generation. But there are mostly in form of traumatic experiences. And then, we learn that these memories could be transferred onto their children, or we could say it as "post generation". In the novel Dora Bruder, such things happen when the author, Patrick Modiano, plays his attribute in composing genres to arrange and transfer his message. The story mainly concerns as the narrator try to find a missing girl named Dora Bruder. She was gone in 1941, or in the moment when Nazi was occupying France. This research aims to discover the relationship between the role of genre on emerging the message, particularly the traumatic ones by using the concept of genre and post-memory. From the analysis we conclude that Modiano use genres to transfer his message traumatic. It exists in form of the impression of absence. From the sensation of absence, he continues to transmit consecutively another impression of hollow, doubt, and hope. For transferring his message and memory, Modiano mixes real documents and his fiction. He manifests them by constructing a story of another person and narrating it from the first-person point of view. He uses this technique to identify himself, because the "shared idea" of one's could be related with another's.
\end{abstract}

\section{Keywords}

Dora Bruder, post memory, memory transmission, genre, traumatic experience 


\section{Introduction}

Parlant l'Holocauste, cela ne veut dire seulement pas qu'on parlant la guerre, mais aussi le traumatisme qui se fossilise au corps des survivants. Et ce n'est pas assez; parce qu'ils certainement ont des enfants, ou on peut en itérer en tant que post générations de l'Holocauste. Il arrive que le sentiment frappant dans " les enfants de la guerre » émerge, même s'ils sont nés après la guerre fût finie. Cité de Eva Hoffman (2004), l'écrivain et en même temps l'un des post générations ; pour elle et les gens qui sont nées après cela, le monde émerge de laquelle et ne vient jamais de l'utérus de ses mères. Elle a de raison, car ils sentent mal de voir des circonstances autour de lui qui étaient en désordre - en fait dès qu'ils juste arrivaient sur la terre. Mais ils ne connaissent jamais la raison de cette condition terrible ; comme Hoffman dit, qu'en tant qu'une fille de survivant qui a été née en 1945, sa première impression était en forme de "réception enfantin ». Cela, elle continue, était un passé inconnu, duquel internalisé profondément mais aussi étrange.

Marianne Hirsch (2001) raconte l'histoire de Susan Sontag en 1973 (On Photography) et Alice Kaplan (French Lesson, 1993) sur leur confrontation avec des mémoires. Sontag était douze en 1945, et Kaplan était huit ou neuf en 1962. Les deux ont vu des photos; des corps, qui s'accumulent et s'empilent - des victimes de la guerre. Desquelles, d'après Sontag, sont "l'inventorie photographique d'horreur ultime » lors de son enfance. Donc, le traumatisme de la guerre, en contexte de l'Holocauste, se place même se rigidifie aux enfants des survivants même lorsqu'ils avaient été née quelques années avant la fin de la guerre. Comme Hoffman (2004) indique, qu'ils sont « les sentinelles de l'Holocauste ».

Même en France, la tragédie devient aussi l'un des événements le plus terrible. Il y a environ 77.000 juifs de France deviennent des victimes pendant l'Occupation de Nazi qui s'est passé en 1940-1945 (Webster, 2011). Dora Bruder est l'un desquelles, qui a perdu en 1941. Un fait-divers concernant la perte de la fille est apparu en Paris-Soir en 1941, ainsi celui est convenu en forme du roman par Patrick Modiano. Ce romancier juste arrive sur la terre en même temps comme Hoffman, en 1945 ou précisément durant la guerre est terminé. Par conséquent, on peut l'appeler aussi comme un post générations. Dora Bruder (1997) n'est seulement l'un des romans qui lui écrit. Il y a d'autres ouvres comme sa première écriture, La Place de l'étoile (1968), et puis La Ronde de nuit (1969), Les Boulevard de ceinture (1972), Rue des Boutiques Obscures (1978). Pour la plupart, ses ouvres montrent les années de la guerre, ou avant qu'il soit né. Son père était français juif, mais sa mère n'était pas (Belgique, pas juive). Néanmoins, ses ouvres dominé par l'histoire de réunion imaginative entre lui et son père (Cooke, 2004).

D'ailleurs, Modiano est aussi une représentation essentielle des post générations. Parmi les productions culturelles consistent les problèmes de la seconde guerre surtout de l'Holocauste en France, Modiano souvent a été cite comme un personnage majeur dans le processus de confrontation avec le passé obscur de l'Occupation (Damamme-Gilbert, 2015). En outre, il devient aussi l'un des écrivains le plus prolifique sur la scène française contemporaine (Rose, 2008). Pour Dora Bruder lui-même, Cooke (2004) l'apprécie aussi comme le travail le plus poignant de lui et possiblement, comme la plus nombreuse cité des textes aux dates. Celui parce que Modiano porte tant des documents historique et biographique dedans là. Il n'oublie jamais également sa fiction ; d'amener le souhaite pour mettre un rendez-vous imaginatif avec son père. Par conséquent, ce roman ne comprend jamais seulement par un genre rigide. On le voit par le processus d'entrelacer entre une autobiographie, autofiction (une fiction de soi), ou même biographie. Damamme-Gilbert (2015) continue que si notre parole sur ses romans vient de seulement un genre rigide, on ne peut jamais rencontrer la plus intense discussion. Elle nous offre que chaque concept diffèrent veut nous aider d'examiner mieux les œuvres de Modiano, dans ce contexte, Dora Bruder.

\section{Les problématiques et les questions de recherche}

Basé sur l'introduction, on ramasse les deux choses le plus important. Le premier, on comprend que Modiano est l'écrivain de post générations de l'Holocauste. Ça veut dire que dans ses écritures, il y a des messages qu'il veut envoyer sur le traumatisme culturelle-soit explicitement, soit implicitement-dont il n'expérience jamais. Il n'avait pas été jamais dans la situation, donc cela devient un sens qui sort en forme de transmission de mémoire de génération avant. Il réitère des impressions et sensations de la guerre, surtout de l'Holocauste.

Le seconde, celui arrive de manière d'exprimer ces impressions et sensations ; et on en connaît par fusionner les archives actuelles qui réellement existe, avec l'imagination de lui-même-la fiction. Comme il dit à l'entretien de Gallimard (2004), il « rêve des morceaux de réalité, ensuite de rassembler tant bien 
que mal dans un livre ». Par suite, il faut employer tous ce qu'il ne se nécessite ni les faits ni la fiction pour traduire ce qu'il sent à ses romans.

Selon les deux problématiques, il arrive deux questions qu'on essaye à répondre sur cette recherche :

a. Comment l'écrivain emploie de fusion des genres pour exprimer le processus de transmission de mémoire?

b. Pourquoi l'écrivain choisi cette manière?

\section{Les objectifs}

Cette recherche a deux buts essentiels : d'expliquer la manière d'employer de fusion des genres sur le processus de transmission de mémoire, et de rendre clair la raison derrière l'usage de cette manière.

\section{Les théories pertinentes}

Robyn Ferrel sur son livre Genre of Philosophy (2002, via Down \& Rulyova, 2015) dit que le genre existe pour la philosophie en tant qu'une question ontologique. Elle continue que le genre est un concept travaillé pour capter le générative a la pensée. Celui est un processus où une pensée nouvelle pourrait représenter quelque chose vieux mais toujours d'être original - la réitérer comme une nouvelle. Ce qui devient le modulateur, d'après Ferrel, et le jeu de répétition et différence. Les deux, ajouté par Dowd (2015), sont le « terrain standard » de théories de genre. Si on en voit de perspective de Bakhtinian, des genres sont des moyens conventionnalisés et partagés pour se servir de langue. Celles-ci pour acquérir des actions déterminent en contexte spécifique (Smedegaard, 2015). La connaissance des genres englobe sur des voix propres et des positionnements que des écrivains et des lecteurs pourraient s'employer et s'assumer. On cherche et utilise tel manière à l'intention de décrire des types des genres qu'on veut en employer.

On cite aussi Damamme-Gilbert (2015), que des genres des textes narratifs se divisent en sous-genres, comme de fiction, biographie, et autobiographie. Le dernier, exprimé par Lejeune que la caractéristique de soi sur le livre d'autobiographie est la question de contrat entre d'auteur et de lecteur. Il y a aussi l'autofiction, un terme présenté par Dubrovsky pour expliquer des fictions de soi. On parle souvent ce genre avec les ouvres de Modiano. Ce qui nous intéresse de ses ouvres est lorsque son narrateur ressemble d'autobiographe, mais en même temps il faut raconter quelques parties de biographies de l'autre. En conséquence, des problèmes des genres arrivent et démarrent la discussion plus tard.

En attendant, le moyen d'expliquer des problèmes par la technique de collage (Nordholt, 2012 via Damamme-Gilbert) ; de placer des documents authentique dans le roman donc en apporte plus au travail documentaire. Simultanément, il en fusionne avec l'imagination de lui-même ; la fiction, pour qu'il transmet des messages. L'un des eux est la question de traumatisme qui lui frappe profondément. En entrelaçant ce message, on l'explique par le concept de post mémoire pour qu'on apprenne ce qui consiste dedans là.

Marianne Hirsch est le premier qui exprime la terminologie de post mémoire en 1997 sur son livre intitulé Family Frames : Photography Narrative and Post Mémoire (Gratton, 2004). Elle décrit que le post mémoire est une forme particulière de mémoire qui est puissant, grâce à ses connections avec des objets ou des ressources. Mais, lesquelles ne s'est pas entremis par la façon de recollection. Il faut employer l'investissement imaginatif et la création pour rendre clair des connections existant. Que les enfants ici sont dominés par des narratives de ses parents qui avaient expériencé un évènement traumatique monumental. Fernanda (2017) amplifie que des mémoires du passé des post générations sont le fondement d'histoire ; par combiner des mémoires des autres (memory of others) avec les imaginations de leurmême. Celles-ci fait sembler qu'ils construisent un corps nouveau pour ses mémoires du passé. Donc, ils génèrent leur œuvre mais aussi une forme de clarification à propos de discours du passé - il s'agit de différences qu'on doit publier. Hirsch (2001) justifie que l'œuvre des post générations exprime l'héritage familial et la transmission de traumatisme culturelle. La curiosité et le désir de post générations pour trouver des expériences de dernier, à mesure qu'ils doivent démasquer la connaissance sur ses parents, devient la relation entre lui et des générations précédentes.

En arrière-plan, je cite « l'inventorie photographique d'horreur ultime » de Susan Sontag lorsqu'elle exprime la sensation de voir quelques photos de l'Holocauste. Expliquant par Gratton (2004), que des photographies en fait sont les ressources fragmentaires et le fond de construction de travail de post mémoire. Elle en regarde comme les certificats de référence ; par se lier le souvenir de la première et seconde génération, ils confirment et maintiennent l'existence de dernier au retardataire. Burgelin (via Gratton, 2004) explique plus étoffer que l'investissement imaginatif et le processus de métaphoriser con- 
duisent des artistes de post génération " génériquement coincé » parmi l'autobiographie, la biographie, et la fiction de soi ou on la connait par le terme « d'autofiction».

\section{Méthode de recherche}

La méthode de cette recherche est divisée en deux étapes primaires. Pour le premier, on concentre d'obtenir des données. Particulièrement, desquels sont en forme de narration dans le roman qui conduit à l'idée de l'usage de fusion des genres pour transmettre le message de post mémoire. Ensuite, on veut en analyser en employant le concept de genre ayant se relie avec le concept de post mémoire de Marianne Hirsch. On les analyse pour trouver ce que l'écrivain veut transmettre et le moyen de cette transmission.

\section{Résultats et discussion}

Tout d'abord, pour qu'il commence de narration, Modiano décide de citer un fait-divers - un fait - sur la perte d'une fille s'appelle Dora Bruder :

Il y a huit ans, dans un vieux journal, Paris-Soir, qui datait du 31 décembre 1941, je suis tombe à la page trois sur une rubrique : "D'hier a aujourd'hui ». Au bas de celle-ci, j'ai lu :

«PARIS

On recherche une jeune fille, Dora Bruder, 15 ans, 1m55, visage ovale, yeux gris-marron, manteau sport gris, pull-over bordeaux, jupe et chapeau bleu marine, chaussures sport marron. Adresser toutes indications à M. et Mme. Bruder, 41 boulevard Ornano, Paris. » (Modiano, 7)

Le narrateur dit que "je suis tombé à la page trois sur une rubrique » donc on pourrait assumer qu'il ne trouve pas ce fait-divers intentionnellement. Il juste l'arrive et continue de le rédiger. Mais, l'avis de recherche lui a "profondément troublé », comme il avoue à interview au Gallimard (2004). L'un de raison est parce qu'il connait bien les endroits autour de ce que la famille Bruder avait habité. Il se frappe en lisant ces endroits, qu'il en distinguait depuis son enfance. Celui forme une relation particulière, inconsciente, bizarre, mais aussi lui fait bouleverser. Il y a des ressemblances que l'écrivain sent entre lui et cette fille Dora, et il s'agit qu'un message de Dora a été envoyé vers lui. Curto (2014) ajoute que ici, Modiano identifie le soi par des « contextes partagés »; une relation d'espace entre lui et Dora Bruder. D'ailleurs, ce fait-divers réel apparu en roman est un exemple de technique de collage (Nordholt, 2012). Le narrateur apporte une preuve d'existant de quelqu'une vrai. Qu'il se frappe aussi par cette découverte, donc il veut chercher tel chose qu'on lui fait frapper en sensation. Donc, il travaille pour tracer la fille perdu et ses parents. Un moment, lorsqu'il considère que les itinéraires et les impressions qu'il garde toujours n'étaient pas au hasard (Modiano, 10), il dit comme-ci :

Peut-être, sans que j'en éprouve encore une claire conscience, étais-je sur la trace de Dora Bruder et de ses parents. Ils étaient la, déjà, en filigrane. (Modiano, 11)

Que le narrateur au moment précis identifie les impressions fugitives qu'il garde par tel d'existence de la trace de Dora Bruder. Il n'y a rien ni de rendez-vous, ni de faire se contacter, mais il en est sûr qu'il existe toujours, cette filigrane de la fille disparu, et aussi ses parents. L'autre occasion qu'il pourrait traverser, quand il veut demander une dérogation au Palais de Justice (Modiano, 16). Ici, une impression ressemble de manière quand il avait cherché son père :

Il m'était arrivé une aventure semblable, vingt ans auparavant. J'avais appris que mon père était hospitalisé à la Pitié-Salpêtrière. Je ne l'avais plus revu depuis la fin de mon adolescence. Alors, j'avais décidé de lui rendre visite à l'improviste. (Modiano, 17)

Le narrateur finalement trouve une raison, une relation; la chose frappant qui lui fait s'identifier à Dora Bruder. Ce qu'il se perd est son père. L'absence de lui explicitement écrit, même il ne le voit jamais depuis la fin de son adolescence. Cela est la sensation quand il avait décidé de "lui rendre visite à l'improviste ». Il y a une forme de commémoration de son père de décrire cette impression. Cooke (2004) ajoute que le sujet «je» joue un rôle vital - la suite est l'un des exemples - en augmentant le sens personnel dans le texte et servant comme le guide de conscience. En outre, il se souvient qu'il avait déjà 
retracé son père ; la même qu'aujourd'hui qu'il passe les jours pour trouver Dora Bruder. Mais, après la trace lui amène a rien, le doute émerge :

Je finissais par douter de l'existence de mon père en passant et repassant devant cette église majestueuse et ces corps de bâtiment irréels, intacts depuis le XVII siècle et qui m'évoquaient Manon Lescault et l'époque ou ce lieu servait de prison aux filles, sous le nom sinistre d'Hôpital General, avant qu'on les déporte en Louisiane. (Modiano, 17-18)

Il démarre de douter "l'existence de son père » parce qu'il n'avait jamais lui rencontré. La narration d'absence se solidifie. Il faut découvrir rien pour commencer à croire que l'absence existe. La dernière phrase du paragraphe même ne légitime rien que le manque de présence :

Impossible de trouver mon père. Je ne l'ai plus jamais revu. (Modiano, 18)

Après il ne trouve personne, dans ce contexte est son père, il commence à se sentir le sentiment de vide. Il y a un creux parce que l'expectation de mettre un rendez-vous encore une fois avec lui ne se passe jamais. Ainsi, il tente de retrouver l'autre cas en tant qu'une forme de négociation avec ce trou. Quand il cherchait la trace de famille Bruder, il redémasque cette sensation, l'identifie parallèlement avec lui :

On se dit qu'au moins les lieux gardent une légère empreinte des personnes qui les ont habités. Empreinte: marque en creux on en relief. Pour Ernest et Cécile Bruder, pour Dora, je dirai : en creux. J'ai ressenti une impression d'absence et de vide, chaque fois que je me suis trouvé dans un endroit où ils avaient vécu. (Modiano, 28-29)

Le narrateur récupère l'impression d'absence et de vide, autant qu'il a fait avant lorsqu'il a essayé de trouver son père. L'émanation lui arrive, en forme de souvenir qu'il ne rencontre jamais. Il ressent les deux sentiments irréels d'aujourd'hui, basé sur sa recherche de la famille du passé. Il toujours sent le même, comme on arrive à celle-ci :

Je me souviens que pour la première fois, j'avais ressenti le vide que l'on éprouve devant ce qui a été détruit, rase net. Je ne connaissais pas encore l'existence de Dora Bruder. Peut-être - mais j'en suis sur - s'est-elle promenée là, dans cette zone, qui m'évoque les rendez-vous d'amour secrets, les pauvres bonheurs perdus. Il flottait encore par ici des souvenirs de campagne, les rues s'appelaient : allée du Puits, allée du Metro, allée des Peupliers, impasse des Chiens. (Modiano, 35)

Par son travail de redécouvrir les endroits de famille Bruder, il succède également d'émerger l'autre sensation que d'absence et de vide : il se construit bien comment Dora Bruder s'est promenée autour des endroits où elle habitait. Lentement, le mémoire de Dora pénètre au narrateur, se fossilise et remplace le sien. Ici, le narrateur sacrifie son entrepôt de mémoire (storage of memory) pour être comblé par l'autre (Fernanda, 2017). Dora avait ce souvenir de se promener aux rues d'où elle habitait, mais le narrateur le reforme pour qu'il s'identifie lui-même. Mais, parmi des sentiments d'absence, de vide, même possiblement de devenir Dora elle-même, le narrateur sent aussi le souhaite qu'il se sauvegarde toujours :

En écrivant ce livre, je lance des appels comme des signaux de phare dont je doute malheureusement qu'ils puissent éclairer la nuit. Mais j'espère toujours. (Modiano, 42)

Il y a une confrontation entre les deux sentiments ; le doute et l'espérance. Cela est un travail pour fermer le grand creux après il ne pourrait pas retrouver ni son père ni Dora et sa famille Bruder. Cité de Douzou (2007), les deux deviennent les «fantômes » qui lui hantent toujours. Il s'agit de fantôme en forme du doute et de l'espérance.

\section{Conclusion}

D'après la discussion avant, on connait qu'il y a la joue de genre dans l'œuvre par l'écrivain de Dora Bruder, Patrick Modiano. Il s'entrelace les documents réel (ex : le fait-divers) ; même il l'employé en tant que l'introduction du roman. Cette pièce de réalité devient une petite partie pour qu'il ramasse des autres morceaux de biographie de Dora Bruder. De plus, il ajoute aussi des nombreux épisodes de lui-même- 
mélange la biographie de Dora avec la sienne. Ou comme Burgelin (via Gratton, 2004) l'explique, que laquelle est une forme de l'investissement imaginatif et le processus de métaphoriser. Ainsi, de cette manière Modiano décrit le message qu'on apprend que celui est dominé par d'absence. Partant de la sensation d'absence, il transmit consécutivement le vide, le doute, mais aussi l'espérance de qu'un jour, il pourrait fixer cette absence par combler le trou profond causé par ce sentiment. Donc, pour transmettre ce message, Modiano utilise le mélange parmi des documents réels et factuels, ses imaginations de fiction, dont il manifeste sur l'histoire de quelqu'une, en racontant au point de vu de premier personnage. Il exploite cette manière pour s'identifier; que quelqu'un ou quelqu'une, dans un épisode particulier de sa vie, a « les contextes partagés » avec l'autre. La répétition de vide, d'absence, de doute, et la métaphore sur l'espérance signe un message profond qu'il veut nous envoyer ; pour que nous comprenons ce qu'il sent en tant que le post génération d'Holocauste.

\section{Références}

Cooke, D. (2004). Hollow Imprints. Journal of Modern Jewish Studies, 3, 131-145.

https://doi.org/10.1080/1472588042000225802

Curto, R. (2014). Narrating via Fragments: Patrick Modiano's Metonymic Style in Dora Bruder.

Contemporary French and Francophone Studies, 18, 352-359.

https://doi.org/10.1080/17409292.2014.938496

Damamme-Gilbert, B. (2015). The Question of Genre in Holocaust Narrative: The Case of Patrick

Modiano's Dora Bruder. In G. Dowd \& N. Rulyova (Eds.), Genre Trajectories Identifying, Mapping,

Projecting (pp. 45-65). Hampshire: Palgrave Macmillan. https://doi.org/10.1057/9781137505484_4

Douzou, C. (2007). Naissance d’un fantôme : dora Bruder de Patrick Modiano. Protée, 35(3), 23-32.

https://doi.org/https://doi.org/10.7202/017476ar

Dowd, G., \& Rulyova, N. (2015). Genre trajectories: Identifying, mapping, projecting. Genre Trajectories:

Identifying, Mapping, Projecting. Hampshire: Palgrave Macmillan.

https://doi.org/10.1057/9781137505484

Fernanda, A. (2007). Transmisi Memori dan Trauma dans Mother Land par Dmetri Kakmi: Kajian

Postmemory. Poetika: Jurnal Ilmu Sastra, 5(2), 82-95.

https://doi.org/https://doi.org/10.22146/poetika.30937

Gallimard. (2004). Rencontre avec Patrick Modiano, à l' occasion de la parution de Dora Bruder. Retrieved from http://www.gallimard.fr/catalog/entretiens/01034347.htm

Gratton, J. (2005). Postmemory, Prememory, Paramemory: The Writing of Patrick Modiano. French

Studies, 59(1), 1-7. https://doi.org/10.1093/fs/kni067

Hirsch, M. (2001). Surviving Images: Holocaust Photographs and the Work of Postmemory. The Yale Journal of Criticism, 14, 5-37. https://doi.org/10.1353/yale.2001.0008

Hoffman, E. (2004). After Such Knowledge: Memory, History, and the Legacy of the Holocaust. London: Vintage Books. https://doi.org/10.1353/fge.2005.0004

Rose, S.-E. (2008). Remembering Dora Bruder: Patrick Modiano's Surrealist Encounter with the

Postmemorial Archive. Postmodern Culture, 18. https://doi.org/10.1353/pmc.0.0017

Schulte Nordholt, A. (2012). Photographie et image en prose dans Dora Bruder de Patrick Modiano.

Neophilologus, 96(4), 523-540. https://doi.org/10.1007/s11061-011-9288-7 
Smedegaard, A. (2015). Constructing a Voice of Authority in Upper Secondary School Essays. In Genre Trajectories: Identifying, Mapping, Projecting. Hampshire: Palgrave Macmillan.

Webster, P. (2011). The Vichy Policy on Jewish Deportation. Retrieved from

http://www.bbc.co.uk/history/worldwars/genocide/jewish_deportation_01.shtml 